\title{
New Noise-Tolerant ZNN Models with Predefined-Time Convergence for Time-Variant Sylvester Equation Solving
}

\author{
Lin Xiao, Yongsheng Zhang, Jianhua Dai, Jichun Li, and Weibing Li
}

\begin{abstract}
Sylvester equation is often applied to various fields such as mathematics and control systems due to its importance. Zeroing neural network (ZNN), as a systematic design method for time-variant problems, has been proved to be effective on solving Sylvester equation in the ideal conditions. In this work, in order to realize the predefined-time convergence of the $\mathrm{ZNN}$ model and modify its robustness, two new noise-tolerant zeroing neural networks (NNTZNNs) are established by devising two novelly constructed nonlinear activation functions (AFs) to find the accurate solution of time-variant Sylvester equation in the presence of various noises. Unlike the original ZNN models activated by known AFs, the proposed two NNTZNN models are activated by two novel AFs, therefore possessing the excellent predefined-time convergence and strong robustness even in the presence of various noises. Besides, the detailed theoretical analyses of the predefinedtime convergence and robustness ability for the NNTZNN models are given by considering different kinds of noises. Simulation comparative results further verify the excellent performance of the proposed NNTZNN models, when applied to online solution of time-variant Sylvester equation.
\end{abstract}

Index Terms-Zeroing neural network (ZNN), finite-time convergence, nonlinear activation function, Sylvester equation, timevariant problems.

\section{INTRODUCTION}

$\mathbf{S}$ OLVING Sylvester equation is often found in mathematics and control theory and applied to solve various important problems such as eigenvalue assignment [1], and image processing [2]. Therefore, it is a crucial issue to solve Sylvester equation by designing various different schemes. Numerical methods were usually used to solve the static Sylvester equation in the past [3]-[14], such as BartelsStewart, and Hessenberg-Schur iteration methods [8]-[14].

This work was supported by the National Natural Science Foundation of China under grants 61866013, 61503152, 61473259, and 61563017; the Natural Science Foundation of Hunan Province of China under grants 2019JJ50478, 18A289, 2016JJ2101, 2018TP1018, 2018RS3065, and 17A173.

L. Xiao is with Hunan Provincial Key Laboratory of Intelligent Computing and Language Information Processing, Hunan Normal University, Changsha 410081, China, and also with the College of Information Science and Engineering, Jishou University, Jishou 416000, China (e-mail: xiaolin860728@163.com).

Y. Zhang is with the College of Information Science and Engineering, Jishou University, Jishou 416000, China. (e-mail: yszhang95@163.com).

J. Dai is with Hunan Provincial Key Laboratory of Intelligent Computing and Language Information Processing, Hunan Normal University, Changsha 410081, China (e-mail: jhdai@ hunnu.edu.cn).

J. Li is with School of Science, Engineering and Design, Teesside University, Middlesbrough TS1 3BX, U.K. (e-mail: j.li@tees.ac.uk).

W. Li is with the Chow Yuk Ho Technology Centre for Innovative Medicine, the Chinese University of Hong Kong, Hong Kong (e-mail: wellbeinglwb@gmail.com).
However, when faced with the time-variant Sylvester equation, numerical methods (such as Hessenberg-Schur method) may be not suitable due to the high complexity and high sampling in each period.

In the past 30 years, neural networks have been extensively studied and applied in related fields such as robotics, automatic control, and image processing [15]-[18]. In view of its superior parallel processing and easy circuit implementation, neural networks were also used to solve the Sylvester equation. For example, the gradient neural network (GNN) was adopted to solve static Sylvester equation effectively. However, for timevariant Sylvester equation, when GNN is employed, it may not be able to converge to its accurately solution, as there exists a large delay error. In [19], Zhang et al. proposed a special class of neural network (termed Zeroing neural network, ZNN) to solve time-variant Sylvester equation, which is able to achieve the exponential convergence. That is to say, the error will gradually approach to zero, as time goes to infinity. Considering the drawback of the ZNN model with infinitetime convergence, which is difficult to meet the requirements in real-time problems solving, a finite-time convergent ZNN (FTCZNN) was proposed and used to solve static and timevariant Sylvester equations [20]-[23]. Furthermore, the upper bound of the finite-time convergence is theoretically calculated in detail [24]-[31]. Nevertheless, the upper bound of the finitetime convergence is closely related to the initial condition of the FTCZNN model [32]-[36]. In other words, different initial conditions of the FTCZNN model will result in different finitetime convergence performance. However, the initial conditions of some practical models are hard to be regulated or even impossible to be evaluated, which can result in performance degradation of the models. In order to address this problem, it is important to propose a new ZNN model with a predefined convergence time that is independent of the initial conditions.

It is worth pointing out that noise disturbance is ubiquitous and unavoidable in real life. When noise disturbance is injected, the above-mentioned GNN, ZNN and FTCZNN models may not be able to converge to the accurate solution of given problems. Therefore, it is also very meaningful to study the robustness of the ZNN model against external noise disturbance, in addition to convergence speed. Currently, some ZNN models with noise tolerance have been proposed and studied to solve various complex problems in front of external noises [37]-[42]. For example, Jin et al. [37]-[40] proposed an integration-enhanced ZNN (IEZNN) model to solve the optimization problem accurately under a variety 
of noise interference. Guo et al. [43] further applied this design method to realize kinematical control of redundant manipulators. However, the convergence speed of the IEZNN model only reaches the exponential convergence, instead of finite-time convergence, not to mention the predefined-time convergence.

Based on the above considerations, we are committed to proposing new ZNN models for solving time-variant Sylvester equation, which not only achieves the predefined-time convergence, but also tolerates various different kinds of noises. To do so, two novel nonlinear functions are skillfully devised to activate the ZNN model to establish two new noise-tolerant ZNN (NNTZNN) models for solving time-variant Sylvester equation. It is noted that the design of two novel nonlinear activation functions (AFs) are inspired by referring to the idea of [33], [34], [36]. As compared with previous ZNN models activated by some existing AFs (e.g., linear AF, bipolar sigmoid $\mathrm{AF}$, power $\mathrm{AF}$, sign-bi-power $\mathrm{AF}$ ) the proposed two NNTZNN model not only have a predefined-time convergence (instead of exponential convergence) but also have a better robustness. The main advantage of the predefined-time convergence is independent to initial states of the NNTZNN model, which can modify the convergence speed greatly. Furthermore, the convergence upper bounds of the proposed NNTZNN models are analytically estimated in theory under different kinds of external noises. Numerical comparison results further verify the superiority of the proposed NNTZNN models to existing ZNN models for time-variant Sylvester equation.

The rest of this paper is organized as follows. For solving online time-variant Sylvester equation, Section II gives the design process of traditional ZNN model and some commonly used AFs. In addition, on basis of known AFs, two novel nonlinear AFs are presented to modify its comprehensive performance. Section III proposes two NNTZNN models based on two nonlinear AFs, and theoretically analyzes the convergence speed as well as robustness in detail. Simulation results are given in Section IV to show the advantages of two NNTZNN models. Section V concludes the paper. Before ending this section, the highlights of this work are summarized as below.

1) Two novel nonlinear activation functions (AFs) are skillfully devised to improve the comprehensive performance of zeroing neural network (ZNN) according to the idea of the predefined time convergence related to nonlinear control systems.

2) Based on these two AFs, two new noise-tolerant zeroing neural networks (NNTZNNs) are developed to solve time-variant Sylvester equation in the presence of various external noises.

3) Compared with the previous ZNN models for timevariant Sylvester equation, the proposed two NNTZNN models not only have the predefined-time convergence performance, but also have the noise-enduring capability against various external disturbances.

4) It is theoretically proved that the finite convergence time for two NNTZNN models to find time-variant Sylvester equation is predefined. Its advantage is that the predefined time can be calculated as a priori and is independent of initial conditions of practical models.
5) It is numerically demonstrated that two NNTZNN models are effective on solving time-variant Sylvester equation under the interference of various noises (such as constant noises, time-dependent bounded noises, time-dependent unbounded noises).

\section{ZNN MODEL AND ACTIVATION FUNCTIONS}

In this part, we first consider the following time-variant Sylvester equation:

$$
A(t) X(t)-X(t) B(t)=-C(t) \in \mathbb{R}^{n \times n},
$$

where $A \in \mathbb{R}^{n \times n}, B \in \mathbb{R}^{n \times n}$ and $C \in \mathbb{R}^{n \times n}$ stand for known time-variant coefficient matrices of appropriate sizes; and $X(t) \in \mathbb{R}^{n \times n}$ stands for an unknown matrix of appropriate size that needs to be obtained. For convenience of presentation, $X^{*} \in \mathbb{R}^{n \times n}$ is used to denote the theoretical solution of (1). In the following, for completeness of this work, the design process of ZNN for time-variant Sylvester equation is first given. Then, we review some commonly-used AFs that were adopted to activate neural models to modify the performance. At last of this section, two novel nonlinear AFs are presented by following the idea of the predefined time convergence.

\section{A. ZNN Model}

First, for solving time-variant Sylvester equation (1), we can define a matrix-valued error function $E(t)$ :

$$
E(t)=A(t) X(t)-X(t) B(t)+C(t) \in \mathbb{R}^{n \times n} .
$$

It is obvious that if each element of the error function $E(t)$ converges to 0 , the corresponding $X(t)$ is what we want to find. That is, solving time-variant Sylvester equation (1) is equivalently transformed into forcing $E(t)$ converging to 0 .

Then, to make the error function (2) decrease to 0 , the following ZNN design formula is employed [21], [22], [26]:

$$
\frac{\mathrm{d} E(t)}{\mathrm{d} t}=-\gamma \Phi(E(t))
$$

where $\Phi(\cdot): \mathbb{R}^{n \times n} \rightarrow \mathbb{R}^{n \times n}$ stands for an activation function array and $\gamma>0$ stands for a known adjustable parameter.

Then, by substituting error function (2) into ZNN design formula (3) and considering the time derivative of the error function $E(t)$ is $\dot{E}(t)=A(t) \dot{X}(t)+\dot{A}(t) X(t)-\dot{X}(t) B(t)-$ $X(t) \dot{B}(t)+\dot{C}(t)$, the following ZNN model for time-variant Sylvester equation (1) is established:

$$
\begin{aligned}
A(t) \dot{X}(t)-\dot{X}(t) B(t) & =\dot{A}(t) X(t)+\dot{X}(t) B(t) \\
& -\gamma \Phi(A(t) X(t)-X(t) B(t) \\
& +C(t))-\dot{C}(t) .
\end{aligned}
$$

For such a ZNN model, which can be regarded as an ordinary differential equation, if an initial value $X(0)$ is given, it can converge to its equilibrium point and output the accurate solution of time-variant Sylvester equation (1). 


\section{B. Commonly-Used AFs}

In the past decade, various different types of activation functions have widely proposed and investigated to modify the performance of neural networks [44]-[47]. These survey results indicate different AFs would lead to different performance of neural models, and most of nonlinear AFs have a positive effect on the convergence speed of neural models. Similarly, for ZNN model (4), choosing a better AF can further modify its comprehensive performance when applied to solving time-variant Sylvester equation (1) even in the presence of external disturbances. Considering the importance of AFs, in this part, several commonly-used AFs are reviewed and presented as follows [44]-[47].

1) linear activation function (LAF): $\phi(x)=x$;

2) bipolar sigmoid activation function (BPAF): $\phi(x)=(1-$ $\exp (-\xi x)) /(1+\exp (-\xi x))$ with $\xi>1$;

3) power activation function (PAF): $\phi(x)=x^{l}$ with $l>3$ indicating an odd integer;

4) power-sigmoid activation function (PSAF):

$$
\phi(x)= \begin{cases}x^{l}, & \text { if }|x| \geq 1, \\ \frac{1+\exp (-\xi)}{1-\exp (-\xi)} \cdot \frac{1-\exp (-\xi x)}{1+\exp (-\xi x)}, & \text { otherwise, }\end{cases}
$$

with $l>3$ indicating an odd integer and $\xi>1$;

5) hyperbolic sine activation function (HSAF): $\phi(x)=$ $(\exp (\xi x)-\exp (-\xi x)) / 2$ with $\xi>1$;

6) sign-bi-power activation function (SBPAF) : $\phi(x)=$ $\left(|x|^{l}+|x|^{1 / l}\right) \operatorname{sgn}(x) / 2$ with $0<l<1$ and $\operatorname{sgn}(\cdot)$ denoting the signum function.

It is worth noting that the above all nonlinear AFs have been used to accelerate the convergence speed of previous $\mathrm{ZNN}$ models and the results are better than that using the linear AF. In addition, if SBPAF is used, finite-time convergence can be realized for ZNN models. However, these nonlinear AFs are only used in the ideal conditions (i.e., no external noises exist).

\section{Two Novel Nonlinear AFs}

As mentioned above, nonlinear AFs can improve the convergence rate even to finite-time convergence performance of ZNN models, but the noise disturbances are essentially not taken into account. That is to say, the above nonlinear AFs activated ZNN models may no longer work effectively when the noise is disturbed. Therefore, on basis of these AFs, inspired by the idea of the predefined-time stability for nonlinear dynamic systems [32]-[34], [36], two novel nonlinear AFs have been developed to activate $\mathrm{ZNN}$ model (4), which can make it not only converge to the equilibrium point in a predefined time, but also tolerate various different external disturbances. Specifically, such two novel nonlinear AFs are presented as follow:

$$
\begin{gathered}
\psi_{1}(x)=\left(a_{1}|x|^{\eta}+a_{2}|x|^{w}\right) \operatorname{sgn}(x)+a_{3} x+a_{4} \operatorname{sgn}(x), \\
\psi_{2}(x)=b_{1} \exp \left(|x|^{p}\right)|x|^{1-p} \operatorname{sgn}(x) / p+b_{2} x+b_{3} \operatorname{sgn}(x),
\end{gathered}
$$

where design parameters $0<\eta<1, w>1, a_{1}>0, a_{2}>0$, $a_{3} \geqslant 0, a_{4} \geqslant 0,0<p<1, b_{1}>0, b_{2} \geqslant 0$, and $b_{3} \geqslant 0$.

In the simulation part, for the purposes of comparison, three typical AFs (i.e., LAF, PSAF, and SPBAF) will be used to activate ZNN model (4) for finding the solution of time-variant Sylvester equation (1) under different noise disturbances.

\section{NNTZNN MODELS AND THEORETICAL ANALYSIS}

In this section, on basis of two novel AFs (5) and (6), two new noise-tolerant ZNN (NNTZNN) models are proposed to solve time-variant Sylvester equation (1). In addition, the detailed theoretical analyses of the predefined-time convergence for two NNTZNN models are discussed in the presence of various different kinds of external disturbances.

\section{A. NNTZNN-1 Model}

In the above section, two novel AFs are presented to modify the comprehensive performance of ZNN model (4) when applied to time-variant Sylvester equation (1) solving. Note that AFs (5) and (6) are standardly scalar-valued functions, while ZNN model (4) is a matrix-valued neural model. Therefore, AFs (5) and (6) are needed to be extended to matrix-valued ones. To do so, we use $\Psi_{1}(x) \in \mathbb{R}^{n \times n}$ to denote the matrixvalued AF array, which is consist of $n \times n$ AF (5). Therefore, when AF array $\Psi_{1}(x) \in \mathbb{R}^{n \times n}$ is used to activate $\mathrm{ZNN}$ model (4), we can obtain the first new noise-tolerant zeroing neural network (NNTZNN) model:

$$
\begin{aligned}
A(t) \dot{X}(t)-\dot{X}(t) B(t) & =\dot{A}(t) X(t)+\dot{X}(t) B(t) \\
& -\gamma \Psi_{1}(A(t) X(t)-X(t) B(t) \\
& +C(t))-\dot{C}(t) .
\end{aligned}
$$

For easy presentation, this model is called the NNTZNN1 model. As compared with ZNN model (4) activated by existing AFs, NNTZNN-1 model (7) has a superior predefinedtime convergence regardless of whether there exist external disturbances, which can be calculated as a priori and is independent of initial conditions of NNTZNN-1 model (7). This feature is important for some practical models where their initial conditions are hard to be regulated or even impossible to be evaluated.

Before the main theoretical results of NNTZNN-1 model (7) are given, the following lemma is first presented as a basis for further discussion [33], [34], [36].

Lemma 1: For a nonlinear dynamic system $\dot{x}(t)=$ $g(x(t), t), t \in[0,+\infty)$ where $g(\cdot)$ denotes a nonlinear function, if there exists a continuous radially unbounded function $V: \mathbb{R}^{n} \rightarrow \mathbb{R}_{+} \cup\{0\}$ such that $V(\zeta)=0$ and any solution $\zeta(t)$ satisfies

$$
\dot{V}(t) \leqslant-\tau V^{\varsigma}(\zeta(t))-\rho V^{\mu}(\zeta(t)),
$$

where parameters $\tau>0, \rho>0,0<\varsigma<1$ and $\mu>1$ are constants, then the predefined convergence time for this system is

$$
T_{\max }=\frac{1}{\tau(1-\varsigma)}+\frac{1}{\rho(\mu-1)} .
$$

Then, we have the following theorem to ensure the predefined-time convergence of NNTZNN-1 model (7) under the ideal conditions. 
Theorem 1: Beginning with a random initial matrix $X(0) \in$ $\mathbb{R}^{n \times n}$, NNTZNN-1 model (7) outputs an accurate solution of time-variant Sylvester equation (1) in a predefined time $t_{\mathrm{c}}$ :

$$
t_{\mathrm{c}} \leqslant \frac{1}{\gamma a_{1}(1-\eta)}+\frac{1}{\gamma a_{2}(w-1)},
$$

where design parameters $\gamma, a_{1}, a_{2}, \eta, w$ are defined as before.

Proof: First, we can conclude that NNTZNN-1 model (7) is equivalent to $\dot{E}(t)=-\gamma \Psi_{1}(E(t))$ with $E(t)$ denoting error function (2), of which the $i, j$ th subsystem is written as

$$
\dot{e}_{i, j}(t)=-\gamma \psi_{1}\left(e_{i, j}(t)\right) \text { with } i, j \in\{1,2, \cdots, n\},
$$

where $e_{i, j}(t)$ and $\dot{e}_{i, j}(t)$ are the $i, j$ th elements of matrices $E(t)$ and $\dot{E}(t)$, respectively.

If this subsystem (8) is proved to be the predefined-time stability, it can be concluded that NNTZNN-1 model (7) is also the predefined-time stability. To prove the predefinedtime stability of the $i, j$ th subsystem (8), a Lyapunov function candidate is first defined as

$$
u(t)=\left|e_{i, j}(t)\right| .
$$

Its time derivative is computed as below:

$$
\dot{u}(t)=\dot{e}_{i, j}(t) \operatorname{sgn}\left(e_{i, j}(t)\right)=-\gamma \psi_{1}\left(e_{i, j}(t)\right) \operatorname{sgn}\left(e_{i, j}(t)\right) .
$$

Since AF (5) is used, we have

$$
\begin{aligned}
\dot{u}(t) & =-\gamma\left(a_{1}\left|e_{i, j}(t)\right|^{\eta}+a_{2}\left|e_{i, j}(t)\right|^{w}+a_{3}\left|e_{i, j}(t)\right|+a_{4}\right) \\
& \leqslant-\gamma\left(a_{1}\left|e_{i, j}(t)\right|^{\eta}+a_{2}\left|e_{i, j}(t)\right|^{w}\right) \\
& =-\gamma\left(a_{1} u^{\eta}(t)+a_{2} u^{w}(t)\right) .
\end{aligned}
$$

By comparing it with the conclusion of Lemma 1, the predefined convergence time of NNTZNN-1 model (7) is directly given by

$$
t_{\mathrm{c}} \leqslant \frac{1}{\gamma a_{1}(1-\eta)}+\frac{1}{\gamma a_{2}(w-1)} .
$$

Since this convergence time $t_{\mathrm{c}}$ is independent of the initial states, NNTZNN-1 model (7) outputs an accurate solution of time-variant Sylvester equation (1) in a predefined time. The proof is thus complete.

Considering that various external disturbances exist during the model hardware implementation, we further investigate the following noise-perturbed NNTZNN-1 model:

$$
\begin{aligned}
A(t) \dot{X}(t)-\dot{X}(t) B(t) & =\dot{A}(t) X(t)+\dot{X}(t) B(t) \\
& -\gamma \Psi_{1}(A(t) X(t)-X(t) B(t) \\
& +C(t))-\dot{C}(t)+\mathrm{Y}(t),
\end{aligned}
$$

where $\mathrm{Y}(t)$ denotes an additive noise. In the following, we mainly study two kinds of additive noises: one is the dynamic bounded vanishing noise, and the other is the dynamic bounded non-vanishing noise.

1) Case 1: When the additive noise $\mathrm{Y}(t)$ is a dynamic bounded vanishing noise, we have the following result for the noise-perturbed NNTZNN-1 model (9).

Theorem 2: If $\mathrm{Y}(t)$ is a dynamic bounded vanishing noise with its $i, j$ th element satisfying $\left|y_{i, j}(t)\right| \leqslant \delta\left|e_{i, j}(t)\right|$ where $\delta \in(0,+\infty)$ and $\left|e_{i, j}(t)\right|$ denotes the absolute value of the $i, j$ th element of error function $E(t)$, beginning with a random initial matrix $X(0) \in \mathbb{R}^{n \times n}$, the noise-perturbed
NNTZNN-1 model (9) outputs an accurate solution of timevariant Sylvester equation (1) in a predefined time $t_{\mathrm{c}}$ :

$$
t_{\mathrm{c}} \leqslant \frac{1}{\gamma a_{1}(1-\eta)}+\frac{1}{\gamma a_{2}(w-1)},
$$

as long as $\gamma a_{3} \geqslant \delta$.

Proof: Similarly, the noise-perturbed NNTZNN-1 model (9) can be simplified as $\dot{E}(t)=-\gamma \Psi_{1}(E(t))+\mathrm{Y}(t)$ with $i, j$ th subsystem formed by

$$
\dot{e}_{i, j}(t)=-\gamma \psi_{1}\left(e_{i, j}(t)\right)+y_{i, j}(t),
$$

where $y_{i, j}(t)$ denotes the $i, j$ th element of matrix $\mathrm{Y}(t)$.

To prove the predefined-time stability of this noiseperturbed subsystem, the following Lyapunov function candidate is chosen:

$$
u(t)=\left|e_{i, j}(t)\right|^{2} .
$$

Besides, $\dot{u}(t)$ is computed as below:

$$
\dot{u}(t)=2 e_{i, j}(t) \dot{e}_{i, j}(t)=2 e_{i, j}(t)\left(-\gamma \psi_{1}\left(e_{i, j}(t)\right)+y_{i, j}(t)\right) .
$$

Since AF (5) is used and $\gamma a_{3} \geqslant \delta$, we have

$$
\begin{aligned}
\dot{u}(t)= & -2 \gamma\left(a_{1}\left|e_{i, j}(t)\right|^{\eta+1}+a_{2}\left|e_{i, j}(t)\right|^{w+1}\right)-2 \gamma a_{4}\left|e_{i, j}(t)\right| \\
& +2\left(e_{i, j}(t) y_{i, j}(t)-\gamma a_{3}\left|e_{i, j}(t)\right|^{2}\right) \\
\leqslant & -2 \gamma\left(a_{1}\left|e_{i, j}(t)\right|^{\eta+1}+a_{2}\left|e_{i, j}(t)\right|^{w+1}\right) \\
& +2\left(\delta\left|e_{i, j}(t)\right|^{2}-\gamma a_{3}\left|e_{i, j}(t)\right|^{2}\right) \\
\leqslant & -2 \gamma\left(a_{1}\left|e_{i, j}(t)\right|^{\eta+1}+a_{2}\left|e_{i, j}(t)\right|^{w+1}\right) \\
= & -2 \gamma\left(a_{1} u^{\frac{\eta+1}{2}}(t)+a_{2} u^{\frac{w+1}{2}}(t)\right) .
\end{aligned}
$$

According to Lemma 1, the predefined time of the noiseperturbed NNTZNN-1 model (9) is calculated as

$$
t_{\mathrm{c}} \leqslant \frac{1}{\gamma a_{1}(1-\eta)}+\frac{1}{\gamma a_{2}(w-1)} .
$$

That is to say, if $\gamma a_{3} \geqslant \delta$, the noise-perturbed NNTZNN1 model (9) outputs an accurate solution of time-variant Sylvester equation (1) in a predefined time under a dynamic bounded vanishing noise.

2) Case 2: When the additive noise $Y(t)$ is a dynamic bounded non-vanishing noise, we have the following result for the noise-perturbed NNTZNN-1 model (9).

Theorem 3: If $\mathrm{Y}(t)$ is a dynamic bounded non-vanishing noise with its $i, j$ th element satisfying $\left|y_{i, j}(t)\right| \leqslant \delta$ where $\delta \in(0,+\infty)$, beginning with a random initial matrix $X(0) \in$ $\mathbb{R}^{n \times n}$, the noise-perturbed NNTZNN-1 model (9) outputs an accurate solution of time-variant Sylvester equation (1) in a predefined time $t_{\mathrm{c}}$ :

$$
t_{\mathrm{c}} \leqslant \frac{1}{\gamma a_{1}(1-\eta)}+\frac{1}{\gamma a_{2}(w-1)},
$$

as long as $\gamma a_{4} \geqslant \delta$.

Proof: Compared with Theorem 2, only the additive noise $\mathrm{Y}(t)$ is different. As a result, according to the subsystem (10), the following Lyapunov function candidate is constructed as

$$
u(t)=\left|e_{i, j}(t)\right|^{2} .
$$

Similarly, $\dot{u}(t)$ is computed as below:

$$
\dot{u}(t)=2 e_{i, j}(t) \dot{e}_{i, j}(t)=2 e_{i, j}(t)\left(-\gamma \psi_{1}\left(e_{i, j}(t)\right)+y_{i, j}(t)\right) .
$$


Since AF (5) is used and $\gamma a_{4} \geqslant \delta$, we have

$$
\begin{aligned}
\dot{u}(t)= & -2 \gamma\left(a_{1}\left|e_{i, j}(t)\right|^{\eta+1}+a_{2}\left|e_{i, j}(t)\right|^{w+1}\right)-2 \gamma a_{3}\left|e_{i, j}(t)\right|^{2} \\
& +2\left(e_{i, j}(t) y_{i, j}(t)-\gamma a_{4}\left|e_{i, j}(t)\right|\right) \\
\leqslant & -2 \gamma\left(a_{1}\left|e_{i, j}(t)\right|^{\eta+1}+a_{2}\left|e_{i, j}(t)\right|^{w+1}\right) \\
& +2\left(\delta\left|e_{i, j}(t)\right|^{2}-\gamma a_{4}\left|e_{i, j}(t)\right|\right) \\
\leqslant & -2 \gamma\left(a_{1}\left|e_{i, j}(t)\right|^{\eta+1}+a_{2}\left|e_{i, j}(t)\right|^{w+1}\right) \\
= & -2 \gamma\left(a_{1} u^{\frac{\eta+1}{2}}(t)+a_{2} u^{\frac{w+1}{2}}(t)\right) .
\end{aligned}
$$

According to Lemma 1, the predefined time of the noiseperturbed NNTZNN-1 model (9) in this case is calculated as

$$
t_{\mathrm{c}} \leqslant \frac{1}{\gamma a_{1}(1-\eta)}+\frac{1}{\gamma a_{2}(w-1)} .
$$

That is to say, if $\gamma a_{4} \geqslant \delta$, the noise-perturbed NNTZNN1 model (9) outputs an accurate solution of time-variant Sylvester equation (1) in a predefined time under a dynamic bounded non-vanishing noise.

\section{B. NNTZNN-2 Model}

In this part, AF (6) is explored to activate ZNN model (4). For obtaining the new neural model, AF (6) has to be extended to matrix-valued one. Similar with $\mathrm{AF}(5)$, we use $\Psi_{2}(x) \in$ $\mathbb{R}^{n \times n}$ to denote the corresponding activation function matrix array of $\psi_{2}(x)$. So, for emphasizing the importance of this activation function, the following new noise-tolerant zeroing neural network (NNTZNN) is presented as below:

$$
\begin{aligned}
A(t) \dot{X}(t)-\dot{X}(t) B(t) & =\dot{A}(t) X(t)+\dot{X}(t) B(t) \\
& -\gamma \Psi_{2}(A(t) X(t)-X(t) B(t) \\
& +C(t))-\dot{C}(t) .
\end{aligned}
$$

For easy presentation, the above new neural model is termed the NNTZNN-2 model. Furthermore, we can obtain the following theoretical result about the predefined-time convergence of NNTZNN-2 model (11).

Theorem 4: Beginning with a random initial matrix $X(0) \in$ $\mathbb{R}^{n \times n}$, NNTZNN-2 model (11) outputs an accurate solution of time-variant Sylvester equation (1) in a predefined time $t_{\mathrm{c}}$ :

$$
t_{\mathrm{c}} \leqslant \frac{1}{\gamma b_{1}},
$$

where design parameters $\gamma$ and $b_{1}$ are defined as before.

Proof: Similarly, we can also conclude that NNTZNN-2 model (11) is equivalent to $\dot{E}(t)=-\gamma \Psi_{2}(E(t))$, of which the $i, j$ th subsystem is written as

$$
\dot{e}_{i, j}(t)=-\gamma \psi_{2}\left(e_{i, j}(t)\right) \text { with } i, j \in\{1,2, \cdots, n\} .
$$

According to Lyapunov theory, for proving the stability of the $i, j$ th subsystem (12), the following Lyapunov function candidate is chosen:

$$
u(t)=\left|e_{i, j}(t)\right| .
$$

In addition, $\dot{u}(t)$ is calculated as below:

$$
\dot{u}(t)=\dot{e}_{i, j}(t) \operatorname{sgn}\left(e_{i, j}(t)\right)=-\gamma \psi_{2}\left(e_{i, j}(t)\right) \operatorname{sgn}\left(e_{i, j}(t)\right) .
$$

Since AF (6) is used, we have

$$
\begin{aligned}
\dot{u}(t) & =-\gamma\left(b_{1} \exp \left(\left|e_{i, j}(t)\right|^{p}\right)\left|e_{i, j}(t)\right|^{1-p} / p+b_{2}\left|e_{i, j}(t)\right|+b_{3}\right) \\
& \leqslant-\gamma b_{1} \exp \left(\left|e_{i, j}(t)\right|^{p}\right)\left|e_{i, j}(t)\right|^{1-p} / p \\
& =-\gamma b_{1} \exp \left(u^{p}(t)\right) u^{1-p}(t) / p .
\end{aligned}
$$

For obtaining the predefined convergence time, we have to calculate $\dot{u}(t) \leqslant-\gamma b_{1} \exp \left(u^{p}(t)\right) u^{1-p}(t) / p$. Therefore, for the $i, j$ th subsystem (12), we have

$$
t_{i, j} \leqslant \frac{1-\exp \left(-u^{p}(0)\right)}{\gamma b_{1}} .
$$

Because $\exp \left(-u^{p}(0)\right)=\exp \left(-\left|e_{i, j}(0)\right|^{p}\right) \in(0,1]$, for NNTZNN-2 model (11), we finally obtain:

$$
t_{\mathrm{c}}=\max \left(t_{i, j}\right) \leqslant \frac{1}{\gamma b_{1}} .
$$

That is to say, the upper bound of the convergence time for NNTZNN-2 model (11) is a constant and independent of the initial states, so NNTZNN-2 model (11) outputs an accurate solution of time-variant Sylvester equation (1) in a predefined time. The proof is thus complete.

Considering that various external disturbances exist during the model hardware implementation, we further investigate the following noise-perturbed NNTZNN-2 model:

$$
\begin{aligned}
A(t) \dot{X}(t)-\dot{X}(t) B(t) & =\dot{A}(t) X(t)+\dot{X}(t) B(t) \\
& -\gamma \Psi_{2}(A(t) X(t)-X(t) B(t) \\
& +C(t))-\dot{C}(t)+\mathrm{Y}(t),
\end{aligned}
$$

where $\mathrm{Y}(t)$ denotes an additive noise. In the following, we also mainly study two kinds of additive noises: one is the dynamic bounded vanishing noise, and the other is the dynamic bounded non-vanishing noise.

1) Case 1: When the additive noise $Y(t)$ is a dynamic bounded vanishing noise, we have the following result for the noise-perturbed NNTZNN-2 model (13).

Theorem 5: If $\mathrm{Y}(t)$ is a dynamic bounded vanishing noise with its $i, j$ th element satisfying $\left|y_{i, j}(t)\right| \leqslant \delta\left|e_{i, j}(t)\right|$ where $\delta \in(0,+\infty)$ and $\left|e_{i, j}(t)\right|$ denotes the absolute value of the $i, j$ th element of error function $E(t)$, beginning with a random initial matrix $X(0) \in \mathbb{R}^{n \times n}$, the noise-perturbed NNTZNN2 model (13) outputs an accurate solution of time-variant Sylvester equation (1) in a predefined time $t_{\mathrm{c}}$ :

$$
t_{\mathrm{c}} \leqslant \frac{1}{\gamma b_{1}},
$$

as long as $\gamma b_{2} \geqslant \delta$.

Proof: First, NNTZNN-2 model (13) is also equivalent to $\dot{E}(t)=-\gamma \Psi_{2}(E(t))+\mathrm{Y}(t)$, and its $i, j$ th subsystem is the same as

$$
\dot{e}_{i, j}(t)=-\gamma \psi_{2}\left(e_{i, j}(t)\right)+y_{i, j}(t) .
$$

To prove the predefined-time stability of this subsystem, the following Lyapunov function candidate is selected:

$$
u(t)=\left|e_{i, j}(t)\right|^{2} .
$$

Besides, $\dot{u}(t)$ is calculated as below:

$$
\dot{u}(t)=2 e_{i, j}(t) \dot{e}_{i, j}(t)=2 e_{i, j}(t)\left(-\gamma \psi_{2}\left(e_{i, j}(t)\right)+y_{i, j}(t)\right) .
$$




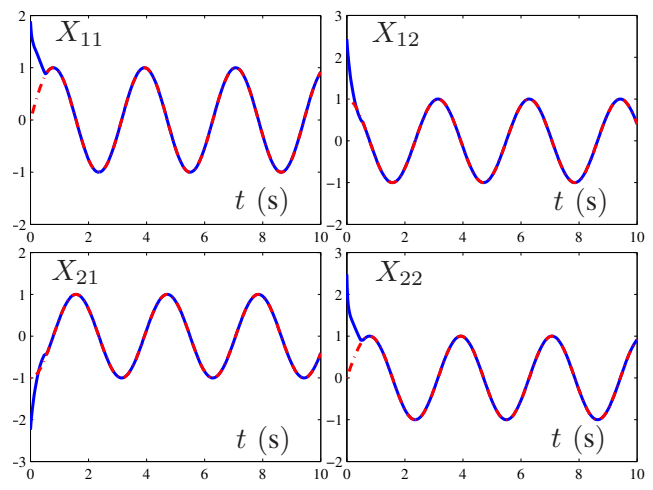

(a) By NNTZNN-1 model (9)

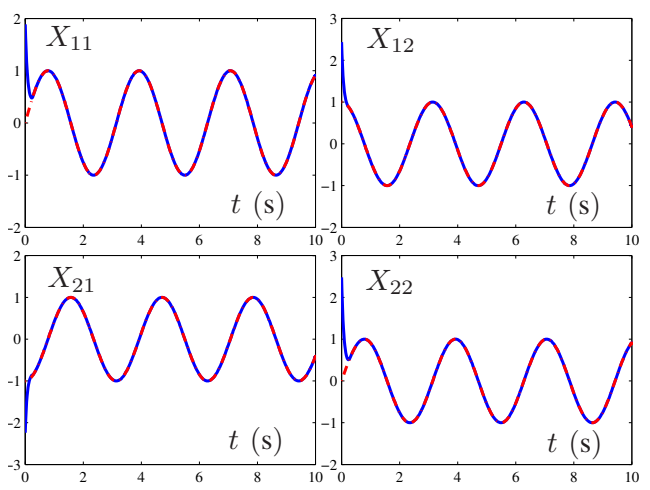

(b) By NNTZNN-2 model (13)

Fig. 1. Transient behavior of state solutions $X(t)$ generated by NNTZNN-1 model (9) and NNTZNN-2 model (13) when solving time-variant Sylvester equation of Example 1 with noise $\mathrm{Y}(\mathrm{t})=0$.

Because AF (6) is used and $\gamma b_{2} \geqslant \delta$, one further can obtain:

$$
\begin{aligned}
\dot{u}(t)= & -2 \gamma b_{1} \exp \left(\left|e_{i, j}(t)\right|^{p}\right)\left|e_{i, j}(t)\right|^{2-p} / p-2 \gamma b_{3}\left|e_{i, j}(t)\right| \\
& +2\left(e_{i, j}(t) y_{i, j}(t)-\gamma b_{2}\left|e_{i, j}(t)\right|^{2}\right) \\
\leqslant & -2 \gamma b_{1} \exp \left(\left|e_{i, j}(t)\right|^{p}\right)\left|e_{i, j}(t)\right|^{2-p} / p \\
& +2\left(\delta\left|e_{i, j}(t)\right|^{2}-\gamma b_{2}\left|e_{i, j}(t)\right|^{2}\right) \\
\leqslant & -2 \gamma b_{1} \exp \left(\left|e_{i, j}(t)\right|^{p}\right)\left|e_{i, j}(t)\right|^{2-p} / p \\
= & -\gamma b_{1} \exp \left(u^{\frac{p}{2}}(t)\right) u^{\frac{2-p}{2}}(t) /(p / 2) .
\end{aligned}
$$

In a same way, the predefined convergence time of NNTZNN2 model (13) can be computed by solving $\dot{u}(t) \leqslant$ $-\gamma b_{1} \exp \left(u^{\frac{p}{2}}(t)\right) u^{\frac{2-p}{2}}(t) /(p / 2)$, and the result is

$$
t_{i, j} \leqslant \frac{1-\exp \left(-u^{\frac{p}{2}}(0)\right)}{\gamma b_{1}} .
$$

As $\exp \left(-u^{\frac{p}{2}}(0)\right)=\exp \left(-\left|e_{i, j}(0)\right|^{\frac{p}{2}}\right) \in(0,1]$, it can also be concluded that:

$$
t_{i, j} \leqslant \frac{1-\exp \left(-u^{\frac{p}{2}}(0)\right)}{\gamma b_{1}} \leqslant \frac{1}{\gamma b_{1}},
$$

which suggests that the upper bound of the convergence time for the $i, j$ th subsystem is a constant and independent of initial states when $\gamma a_{4} \geqslant \delta$. Therefore, if $\gamma a_{4} \geqslant \delta$, the noiseperturbed NNTZNN-2 model (13) outputs an accurate solution of time-variant Sylvester equation (1) in a predefined time under a dynamic bounded vanishing noise. In addition, the predefined convergence time of NNTZNN-2 model (13) is

$$
t_{\mathrm{c}}=\max \left(t_{i, j}\right) \leqslant \frac{1}{\gamma b_{1}} .
$$

The proof is thus complete.

2) Case 2: When the additive noise $\mathrm{Y}(t)$ is a dynamic bounded vanishing noise, we have the following result for the noise-perturbed NNTZNN-2 model (13).

Theorem 6: If $\mathrm{Y}(t)$ is a dynamic bounded non-vanishing noise with its $i, j$ th element satisfying $\left|y_{i, j}(t)\right| \leqslant \delta$ where $\delta \in(0,+\infty)$, beginning with a random initial matrix $X(0) \in$ $\mathbb{R}^{n \times n}$, the noise-perturbed NNTZNN-2 model (13) outputs an accurate solution of time-variant Sylvester equation (1) in a predefined time $t_{\mathrm{c}}$ :

$$
t_{\mathrm{c}} \leqslant \frac{1}{\gamma b_{1}}
$$

as long as $\gamma b_{3} \geqslant \delta$.

Proof: Similar to Theorem 5, the Lyapunov function candidate $u(t)=\left|e_{i, j}(t)\right|^{2}$ is first chosen for the $i, j$ th subsystem of NNTZNN-2 model (13), and $\dot{u}(t)$ is computed as follows:

$$
\dot{u}(t)=2 e_{i, j}(t) \dot{e}_{i, j}(t)=2 e_{i, j}(t)\left(-\gamma \psi_{2}\left(e_{i, j}(t)\right)+y_{i, j}(t)\right) .
$$

Since AF (6) is used and $\gamma b_{3} \geqslant \delta$, one can obtain:

$$
\begin{aligned}
\dot{u}(t)= & -2 \gamma b_{1} \exp \left(\left|e_{i, j}(t)\right|^{p}\right)\left|e_{i, j}(t)\right|^{2-p} / p-2 \gamma b_{2}\left|e_{i, j}(t)\right|^{2} \\
& +2\left(e_{i, j}(t) y_{i, j}(t)-\gamma b_{3}\left|e_{i, j}(t)\right|\right) \\
\leqslant & -2 \gamma b_{1} \exp \left(\left|e_{i, j}(t)\right|^{p}\right)\left|e_{i, j}(t)\right|^{2-p} / p \\
& +2\left(\delta\left|e_{i, j}(t)\right|^{2}-\gamma b_{3}\left|e_{i, j}(t)\right|^{2}\right) \\
\leqslant & -2 \gamma b_{1} \exp \left(\left|e_{i, j}(t)\right|^{p}\right)\left|e_{i, j}(t)\right|^{2-p} / p \\
= & -\gamma b_{1} \exp \left(u^{\frac{p}{2}}(t)\right) u^{\frac{2-p}{2}}(t) /(p / 2) .
\end{aligned}
$$

Similar to the proof process of Theorem 5, we can also conclude that the predefined convergence time for NNTZNN-2 model (13) in this case is

$$
t_{\mathrm{c}} \leqslant \frac{1}{\gamma b_{1}} .
$$

Hence, if $\gamma b_{3} \geqslant \delta$, the noise-perturbed NNTZNN-2 model (13) outputs an accurate solution of time-variant Sylvester equation (1) in a predefined time under a dynamic bounded vanishing noise. The proof is thus complete.

\section{ILlustrative Verification}

In Section III, two NNTZNN models and the corresponding noise-perturbed ones [i.e., NNTZNN-1 model (9) and NNTZNN-2 model (13)] are proposed for solving the timevariant Sylvester equation (1). Different from the previous existing AFs, when AF (5) and AF (6) are used to activate $\mathrm{ZNN}$, the predefined-time convergence can be achieved even if there are noise interruptions. In addition, the predefined-time convergence analyses are provided according to different kinds of noises. In this section, to verify the superior performance of the proposed neural models, three different time-variant Sylvester equation examples are used to test the efficiency. 


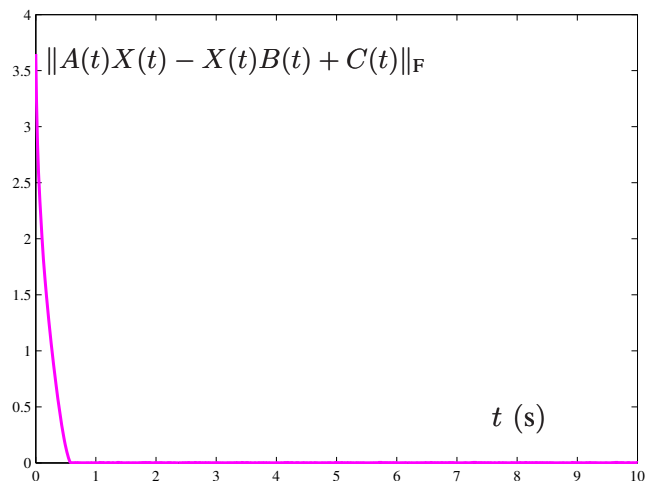

(a) By NNTZNN-1 model (9)

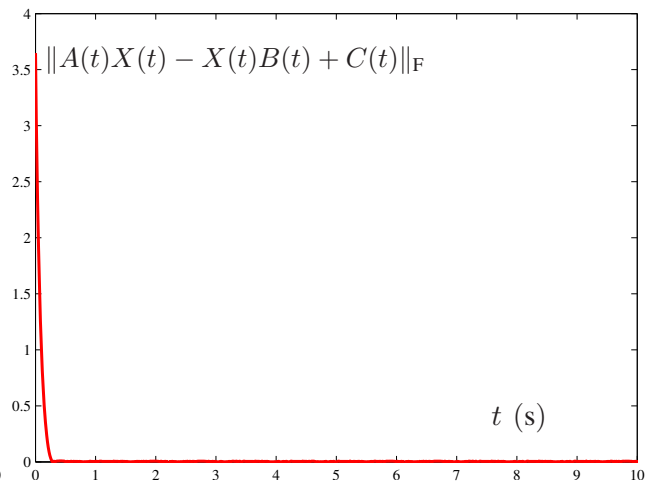

(b) By NNTZNN-2 model (13)

Fig. 2. Transient behavior of residual errors $\|A(t) X(t)-X(t) B(t)+C(t)\|_{\mathrm{F}}$ generated by NNTZNN-1 model (9) and NNTZNN-2 model (13) when solving time-variant Sylvester equation of Example 1 with noise $\mathrm{Y}(\mathrm{t})=0$.

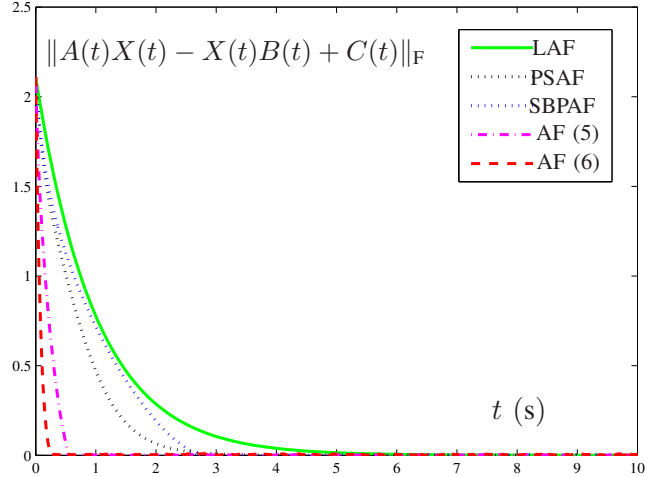

(a) With noise $\mathrm{Y}(\mathrm{t})=0$

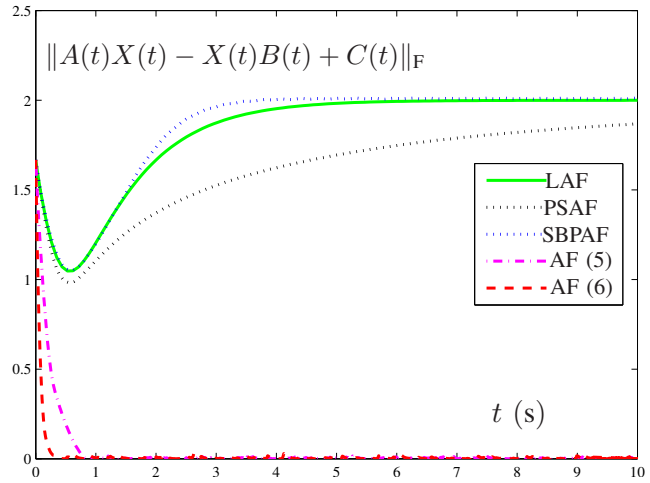

(c) With noise $\mathrm{Y}(\mathrm{t})=1$

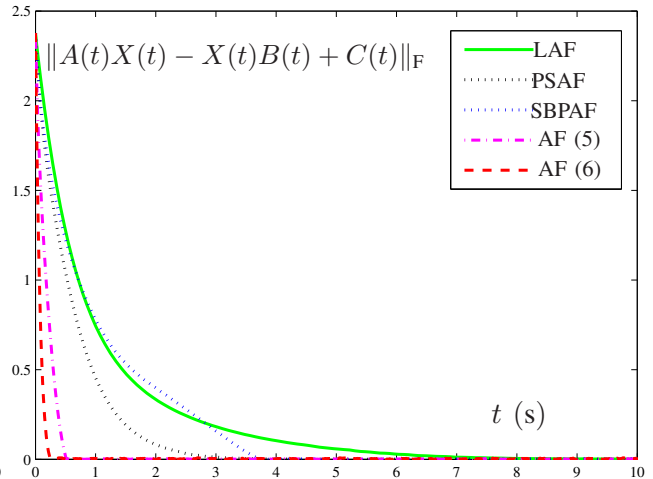

(b) With noise $\mathrm{Y}(\mathrm{t})=0.45\left|e_{i, j}\right|$

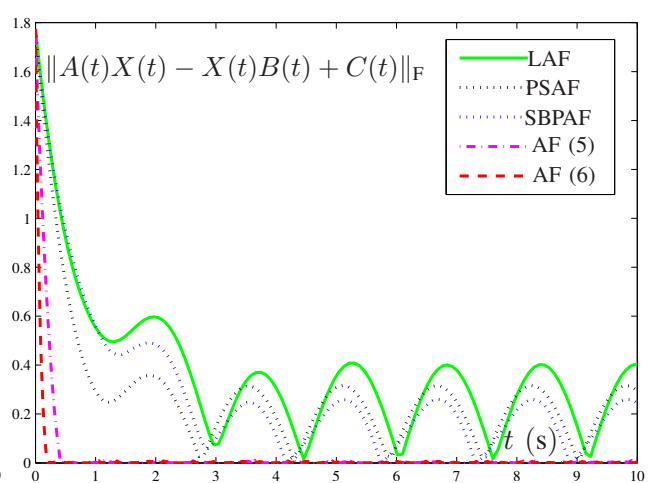

(d) With noise $\mathrm{Y}(\mathrm{t})=0.45 \cos (2 t)$

Fig. 3. Transient behavior of residual errors $\|A(t) X(t)-X(t) B(t)+C(t)\|_{\mathrm{F}}$ synthesized by NNTZNN-1 model (9) activated by AF (5), NNTZNN-2 model (13) activated by AF (6) and ZNN model (4) activated by LAF, PSAF, and SBPAF under different kinds of noises $Y(t)$.

\section{A. Example 1}

Without loss of generality, design parameters are set as $\gamma=a_{1}=a_{2}=a_{3}=a_{4}=b_{1}=b_{2}=b_{3}=1, \eta=$ $p=0.25, w=4$ and the coefficient matrices of time-variant Sylvester equation (1) as follows:

$A(t)=\left[\begin{array}{cc}\sin (2 t) & \cos (2 t) \\ -\cos (2 t) & \sin (2 t)\end{array}\right], B(t)=0$, and $C(t)=-I$.

Obviously, the predefined time of NNTZNN-1 model (9) for solving the time-variant Sylvester equation (1) can be calculated as $t_{c}=5 / 3 \approx 1.67 \mathrm{~s}$, and the one for NNTZNN2 model (13) can be calculated as $t_{c}=1 \mathrm{~s}$. In addition, the theoretical solution $X^{*}(t)$ of the given example can be calculated as

$$
X^{*}(t)=\left[\begin{array}{cc}
\sin (2 t) & -\cos (2 t) \\
\cos (2 t) & \sin (2 t)
\end{array}\right],
$$

which can be used as a criterion for measuring the correctness of each model to solve the time-variant Sylvester equation (1). First, NNTZNN-1 model (9) and NNTZNN-2 model (13) models are used to solve the time-variant Sylvester equation (1) problem without noises [i.e., $Y(t)=0$ ], and the main simulation results are plotted in Figs. 1-2. When AF (5) is activated, the state solution $X(t)$ of NNTZNN-1 model (9) for 


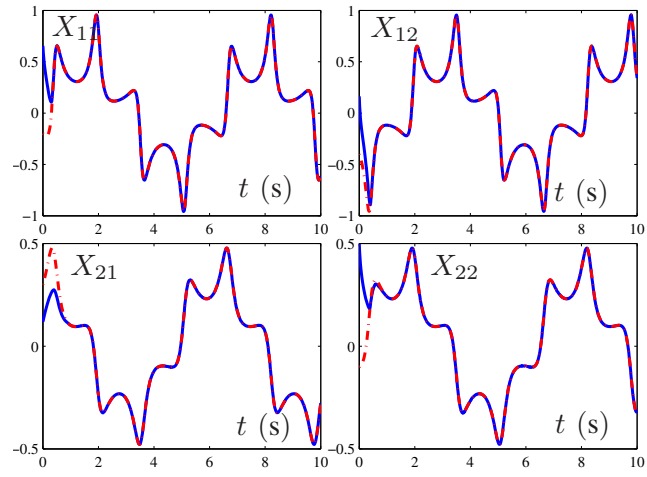

(a) By NNTZNN-1 model (9)

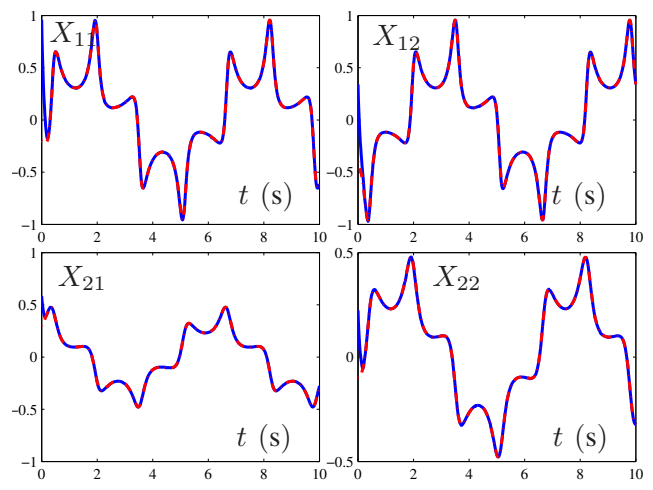

(b) By NNTZNN-2 model (13)

Fig. 4. Transient behavior of state solutions $X(t)$ generated by NNTZNN-1 model (9) and NNTZNN-2 model (13) when solving time-variant Sylvester equation of Example 2 with noise $\mathrm{Y}(\mathrm{t})=1$.

time-variant Sylvester equation is plotted in Fig. 1(a). From it, we can see that the blue solid line coincides with the red dotted line in a very short time, where the blue solid line represents each element of the state solution $X(t)$ from the starting point $X(0) \in[-3,3]^{2 \times 2}$, while the red dashed line represents each element of the theoretical solution $X(t)$. When $\mathrm{AF}(6)$ is activated, the state solution $X(t)$ of NNTZNN-2 model (13) for time-variant Sylvester equation is plotted in Fig. 1(b), which also show that the convergence time required is also very short for coinciding between the blue solid line and the red dotted line.

In addition, the residual errors $\| A(t) X(t)-X(t) B(t)+$ $C(t) \|_{\text {F }}$ synthesized by NNTZNN-1 model (9) and NNTZNN2 model (13) are plotted in Fig. 2. As shown in Fig. 2(a), the residual error of NNTZNN-1 model (9) converges to zero about 0.6 seconds. This means that NNTZNN-1 model (9) only needs about 0.6 seconds to solve time-variant Sylvester equation of Example 1 accurately, and this convergence time satisfies the requirement of the predefined time $t_{c} \leq 1.67$ seconds. Besides, as shown in Fig. 2(b), the residual error of NNTZNN-2 model (13) converges to zero in a shorter time (about 0.3 seconds), which also satisfies the requirement of the predefined time $t_{c} \leq 1$ seconds.

For comparison purposes, ZNN model (4) activated by other AFs [such as LAF, PSAF and SBPAF] are also used to solve time-variant Sylvester equation of Example 1 under different noises, and all comparison results are shown in Fig. 3. If noise $Y(t)=0$, all residual errors can converge to 0 , but NNTZNN2 model (13) has the fastest convergence rate (approximately 0.3 seconds). Followed by NNTZNN-1 model (9), the time required for the residual error converging to zero is about 0.6 seconds), while ZNN model (4) activated by LAF, PSAF, and SBPAF takes longer time to converge to zero (i.e., using SBPAF takes about 2.8 seconds, using PSAF needs about 3 seconds, and using LAF takes about 6 seconds). The results verify the advantages of the proposed two neural models for time-variant Sylvester equation in the presence of no noise.

When the external disturbance is a dynamic bounded vanishing noise $Y(t)=0.45\left|e_{i, j}\right|$, comparison results about the residual errors are shown in Fig. 3(b). From it we can see that the convergence time for NNTZNN-1 model (9) and
NNTZNN-2 model (13) seems to be unchanged, and the others are correspondingly slower, as compared with the results of Fig. 3(a). When the external disturbance is a constant noise $Y(t)=1$, the corresponding residual errors are plotted in Fig. 3(c), which demonstrates residual errors of NNTZNN1 model (9) and NNTZNN-2 model (13) can still converge to zero quickly, while the residual errors activated by LAF, PSAF, SBPAF gradually tends to a stable non-zero value that is usually related with the external disturbance. This means that ZNN model (4) activated by LAF, PSAF, and SBPAF may be no longer effective in the presence of a constant noise, when applied to time-variant Sylvester equation solving. However, the proposed two NNTZNN models can still solve the time-varying Sylvester equation quickly and accurately. When the external disturbance is a dynamic bounded nonvanishing noise noise $Y(t)=0.45 \cos (2 t)$, the corresponding residual error convergence is shown in Fig. 3(d). It can be seen from Fig. 3(d) that the residual errors of NNTZNN-1 model (9) and NNTZNN-2 model (13) can still rapidly drop to zero in a short time, while the residual errors of ZNN model (4) activated by LAF, PSAF, SBPAF always fluctuate all the time. In a word, the superiority of NNTZNN-1 model (9) and NNTZNN-2 model (13) is firmly validated in the presence of various external noises.

\section{B. Example 2}

To further validate the superiority of the proposed two NNTZNN models, the time-variant Sylvester equation coming from [20] is considered, and its coefficients are described by

$$
A(t)=\left[\begin{array}{cc}
\sin (4 t) & \cos (4 t) \\
-\cos (4 t) & \sin (4 t)
\end{array}\right], B(t)=\left[\begin{array}{ll}
2 & 0 \\
0 & 3
\end{array}\right],
$$

and

$$
C(t)=\left[\begin{array}{cc}
\sin (t) & \cos (t) \\
-\cos (t) & \sin (t)
\end{array}\right] .
$$

For consistency, in this example, design parameters of all ZNN models are the same with these of Example 1. According to the theoretical analysis, when NNTZNN-1 model (9) is hired to solve the above time-variant Sylvester equation, it will converge to the theoretical solution within the predefined 


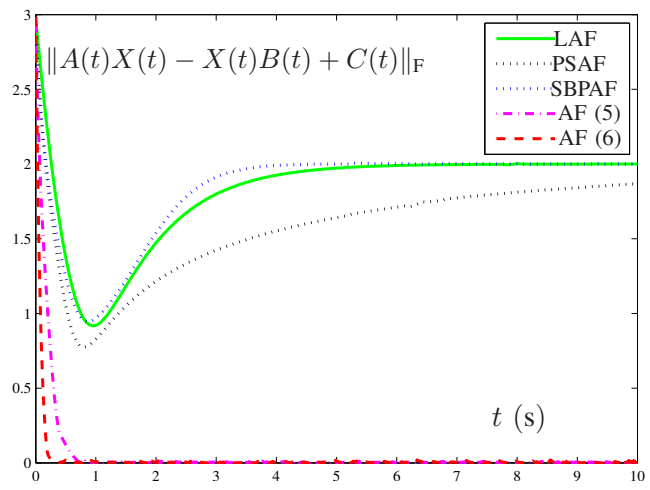

(a) With noise $\mathrm{Y}(\mathrm{t})=1$

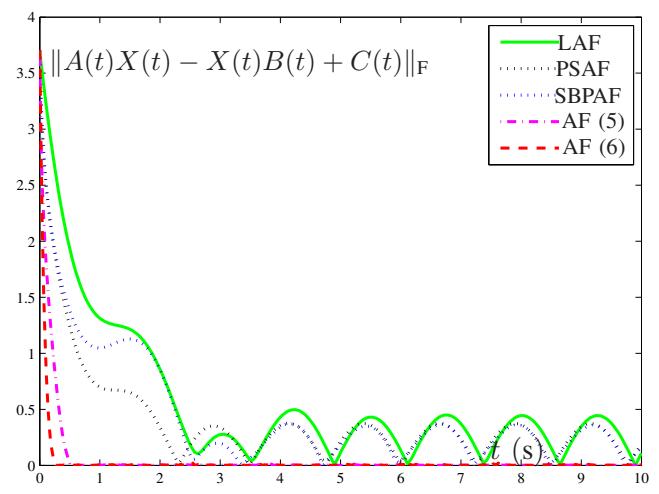

(c) With noise $\mathrm{Y}(\mathrm{t})=0.6 \cos (2.5 t)$

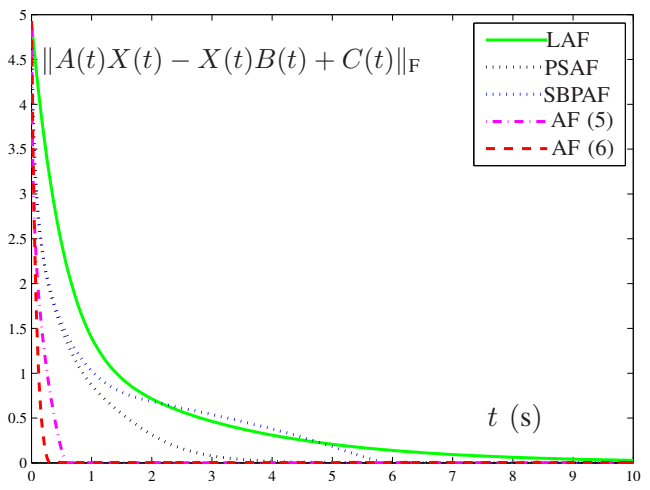

(b) With noise $\mathrm{Y}(\mathrm{t})=0.6\left|e_{i, j}\right|$

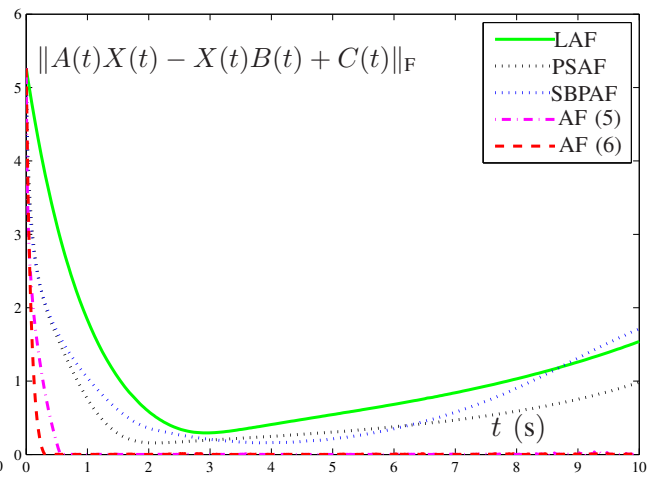

(d) With noise $\mathrm{Y}(\mathrm{t})=0.125 \exp (0.2 t)$

Fig. 5. Transient behavior of residual errors $\|A(t) X(t)-X(t) B(t)+C(t)\|_{\mathrm{F}}$ synthesized by NNTZNN-1 model (9), NNTZNN-2 model (13) and ZNN model (4) activated by LAF, PSAF, and SBPAF under different kinds of noises $Y(t)$ with $\gamma=1$.

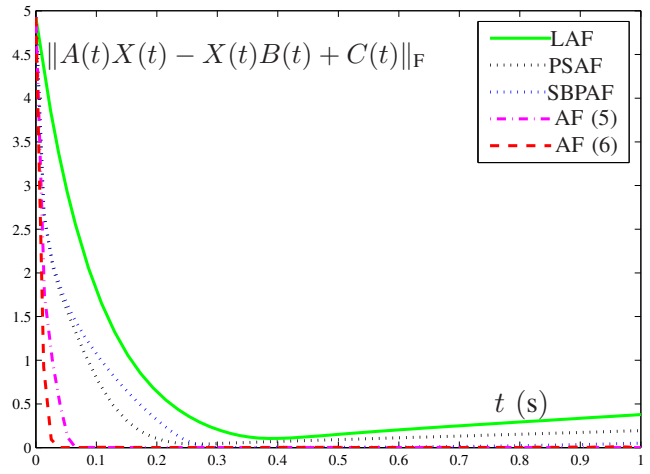

(a) With noise $\mathrm{Y}(\mathrm{t})=2.1 t$ and $\gamma=10$

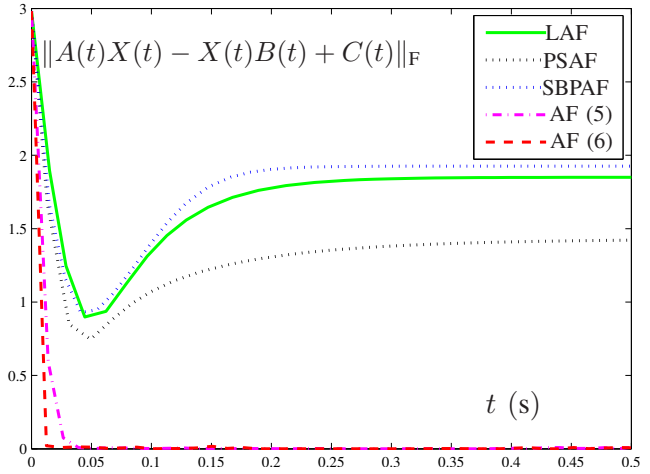

(b) With noise $\mathrm{Y}(\mathrm{t})=18.5$ and $\gamma=20$

Fig. 6. Transient behavior of residual errors $\|A(t) X(t)-X(t) B(t)+C(t)\|_{\mathrm{F}}$ synthesized by NNTZNN-1 model (9), NNTZNN-2 model (13) and ZNN model (4) activated by LAF, PSAF, and SBPAF under different kinds of noises $Y(t)$ with $\gamma=10$ and $\gamma=20$.

time $t_{c}=1.67$ seconds, and when the NNTZNN-2 model (13) is hired, the corresponding predefined time $t_{c}$ equals to 1 second.

First, NNTZNN-1 model (9) and NNTZNN-2 model (13) are employed to solve the above time-variant Sylvester equation in the presence of constant noise $Y(t)=1$, and the corresponding transient behavior of state solutions is plotted in Fig. 4. From Fig. 4(a), it can be observed that each element of state solution $X(t)$ for NNTZNN-1 model (9) from a randomly starting point $X(0)$ coincides with the one of the theoretical solution quickly in predefined time 1 second. In addition, from Fig. 4(b), it follows that the state solutions $X(t)$ of NNTZNN-2 model (13) can converge to the theoretical solution $X^{*}(t)$ under the same conditions in a shorter predefined time (about 0.3 seconds). Note that, in this situation, the convergence time of NNTZNN-1 model (9) and NNTZNN-2 model (13) can satisfy the requirement of the theoretically-computed predefined time.

Fig. 5 shows some comparison results of the residual errors solved by the proposed two NNTZNN models and ZNN model (4) activated by existing AFs under different external noise disturbance (including the situation of constant noise $Y(t)=1$ ). First, Fig. 5(a) shows the results in the presence of constant noise $Y(t)=1$, from which, the residual errors generated 


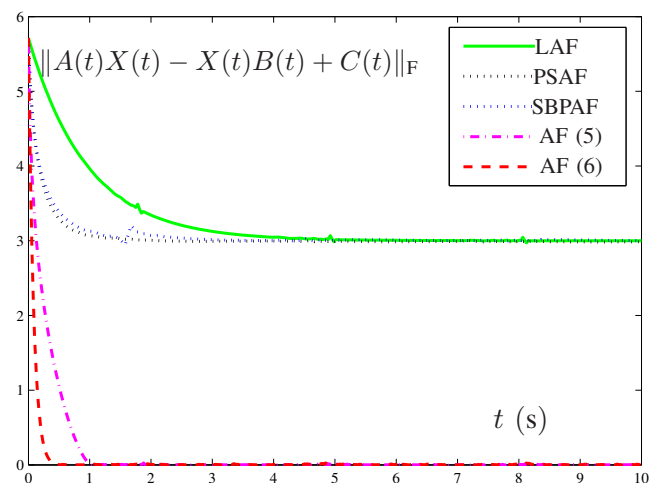

(a) With noise $\mathrm{Y}(\mathrm{t})=1$

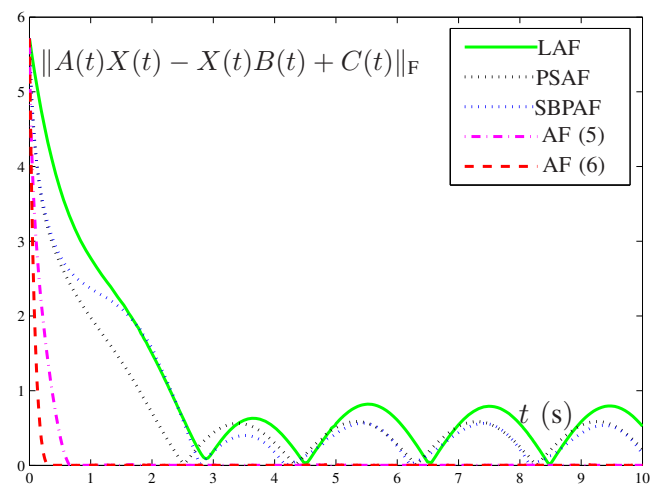

(c) With noise $\mathrm{Y}(\mathrm{t})=0.5 \sin (1.6 t)$

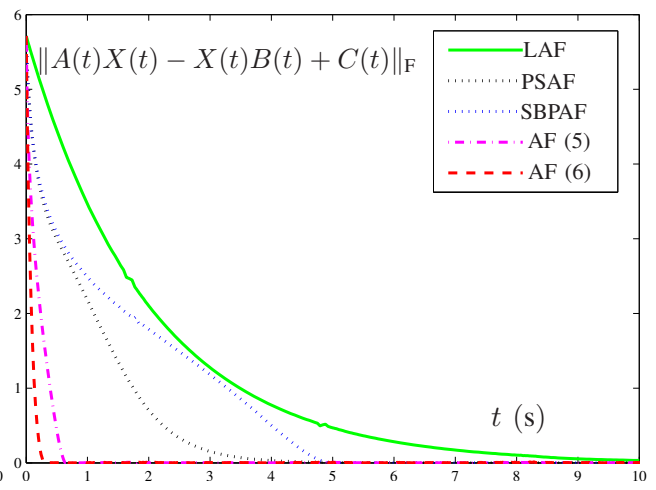

(b) With noise $\mathrm{Y}(\mathrm{t})=0.5\left|e_{i, j}\right|$

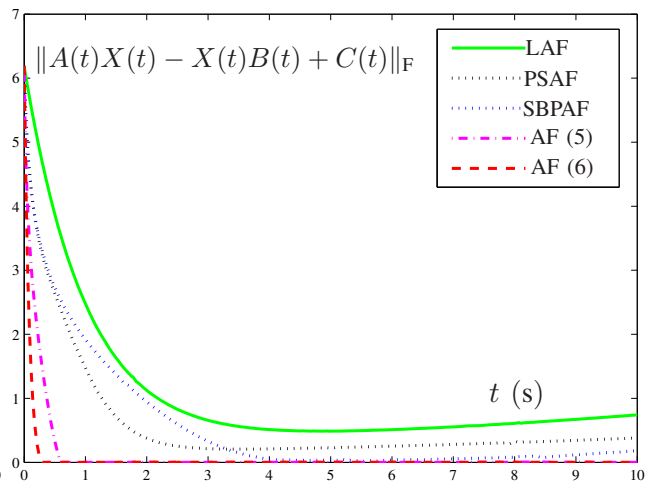

(d) With noise $\mathrm{Y}(\mathrm{t})=0.1 \exp (0.1 t)$

Fig. 7. Transient behavior of residual errors $\|A(t) X(t)-X(t) B(t)+C(t)\|_{\mathrm{F}}$ synthesized by NNTZNN-1 model (9), NNTZNN-2 model (13) and ZNN model (4) activated by LAF, PSAF, and SBPAF under different kinds of noises $Y(t)$ with $\gamma=1$.

by NNTZNN-1 model (9) and NNTZNN-2 model (13) can converge to 0 with the predefined time, while generated by ZNN model (4) activated by existing AFs cannot converge to zero over time. Fig. 5(b) shows that when a fading noise is added, there is a delay for ZNN model (4) activated by existing AFs, as compared to the results of Fig. 3(a), while there is no delay in NNTZNN-1 model (9) and NNTZNN-2 model (13). Besides, time-variant bounded noise $\mathrm{Y}(\mathrm{t})=0.6 \cos (2.5 t)$ and time-variant unbounded noise $\mathrm{Y}(\mathrm{t})=0.125 \exp (0.2 t)$ are considered, and the corresponding comparison results are plotted in Fig. 5(c) and (d), respectively. As seen from such two subfigures, the residual errors of NNTZNN-1 model (9) and NNTZNN-2 model (13) can converge to zero in a predefined time, while ZNN model (4) activated by existing AFs cannot converge to zero all the time, which means that using existing AFs [such as LAF, PSAF, and SBPAF] may no longer be suitable for solving time-variant Sylvester equation (1) when the time-variant noise is injected. In contrast, NNTZNN-1 model (9) and NNTZNN-2 model (13) can still solve the timevariant Sylvester equation accurately within a predefined time.

It is worth noting that the design parameter $\gamma$ has an important impact on the solution process of the $\mathrm{ZNN}$ models. A time-variant non-vanishing noise $\mathrm{Y}(\mathrm{t})=2.1 t$ and a large constant noise $\mathrm{Y}(\mathrm{t})=18.5$ are both considered when the values of the design parameter $\gamma$ are adjusted to 10 and 20, respectively. The corresponding comparison results are described in Fig. 6(a) and (b), respectively. Fig. 6(a) demonstrates that the residual errors of NNTZNN-1 (9) and NNTZNN-2 models (13) can converge to zero. The convergence time for NNTZNN-1 model (9) is reduced to about 0.06 seconds, and for NNTZNN-2 model (13) is reduced to about 0.05 seconds. Considering $\gamma=10$, the predefined convergence time for NNTZNN-1 (9) and NNTZNN-2 model (13) is computed as 0.16 and 0.1 respectively. Obviously, both NNTZNN-1 model (9) and NNTZNN-2 model (13) satisfy the predefined time convergence in the presence of $\mathrm{Y}(\mathrm{t})=2.1 t$ when $\gamma=10$. However, the residual errors of ZNN model (4) activated by existing AFs shown in Fig. 6(a) have a certain error and cannot converge to zero. This conclusion is also demonstrated by Fig. 6(b) conducted in the presence of a large constant noise $\mathrm{Y}(\mathrm{t})=18.5$ and $\gamma=20$.

\section{Example 3}

In this example, a 3-dimensional time-variant Sylvester equation is considered with coefficients being

$$
\begin{aligned}
& A(t)=\left[\begin{array}{ccc}
2+\sin (2 t) & \cos (2 t) & \cos (2 t) / 2 \\
\cos (2 t) & 2+\sin (2 t) & \cos (2 t) \\
\cos (2 t) / 2 & \cos (2 t) & 2+\sin (2 t)
\end{array}\right], \\
& B(t)=0 \in \mathbb{R}^{3 \times 3}, \text { and } C(t)=-I \in \mathbb{R}^{3 \times 3} .
\end{aligned}
$$

The value of the design parameters is consistent with the previous two examples. NNTZNN-1 model (9) and NNTZNN-2 model (13) are hired to solve the above time-variant Sylvester equation in the presence of four different kinds of noise 
with $\gamma=1$, and the corresponding transient behavior of residual errors is plotted in Fig. 7. As seen from Fig. 7, one can found that all residual errors of NNTZNN-1 model (9) and NNTZNN-2 model (13) can converge to 0 within 1 second, which satisfying the predefined-time requirement [i.e., $t_{c}=1.67 \mathrm{~s}$ for NNTZNN-1 model (9) and $t_{c}=1$ $\mathrm{s}$ for NNTZNN-2 model (13)]. In contrast, when external noise $\mathrm{Y}(\mathrm{t})=1, \mathrm{Y}(\mathrm{t})=0.5 \sin (1.6 t)$ or $\mathrm{Y}(\mathrm{t})=0.1 \exp (0.1 t)$ is present, the residual errors of $\mathrm{ZNN}$ model (4) activated by LAF, PSAF and SBPAF cannot converge to 0 . When a fading noise $\mathrm{Y}(\mathrm{t})=0.5\left|e_{i, j}\right|$ is present, all residual errors can converge to 0 , but the convergence time of two NNTZNN models' residual errors is much shorter, as compared with ZNN model (4) activated by LAF, PSAF and SBPAF.

In summary, according to the above comparison result$\mathrm{s}$, it follows that, as compared with ZNN model (4) activated by LAF, PSAF and SBPAF, NNTZNN-1 model (9) and NNTZNN-2 model (13) have superior predefined-time convergence and noise-tolerant performance when applied to time-variant Sylvester equation (1) solving in the presence of various kinds of external disturbances.

\section{Conclusion}

By adopting two nonlinear activation functions (AFs), two new noise-tolerant zeroing neural networks (NNTZNNs) are established to solve time-variant Sylvester equation under various external disturbances. Compared with the ZNN model activated by existing AFs for time-variant Sylvester equation, such two NNTZNN models have superior the predefinedtime convergence and noise-tolerant performance. In addition, the related theorems are rigorously analyzed under no noise, dynamic bounded vanishing noise and dynamic bounded nonvanishing noise. Comparison results further show that the proposed two NNTZNN models converge to the accurate solution of the time-variant Sylvester equation in regardless of whether there exist external noises, while the ZNN model activated by LAF, PSAF and SBPAF cannot converge to the accurate solution under the same conditions. It is worth noting that this is the first co-design of the predefined-time convergence and the noise-tolerant performance for ZNN to solve time-variant Sylvester equation, making it have the better performance in terms of convergence speed and robustness in ZNN field. However, the proposed two NNTZNN models have a relatively high complexity, as compared with the $\mathrm{ZNN}$ model activated by other existing AFs. The future work may optimize the structure of the NNTZNN models and further extend them to some real engineering applications.

\section{REFERENCES}

[1] S. Brahma and B. Datta, "An optimization approach for minimum norm and robust partial quadratic eigenvalue assignment problems for vibrating structures," J. Sound Vib., vol. 324, no. 3-5, pp. 471-489, 2009.

[2] D. Calvetti and L. Reichel, "Application of ADI iterative methods to the restoration of noisy images," SIAM J. Matrix Anal. Appl., vol. 17, no. 1, pp. 165-186, 1996.

[3] L. Bao, Y. Lin, and Y. Wei, "A new projection method for solving large Sylvester equations," Appl. Numer. Math., vol. 57, no. 5, pp. 521-532, 2007.

[4] H. Diao, H. Xian, and Y. Wei, "Mixed, componentwise condition numbers and small sample statistical condition estimation of Sylvester equations," Numer Linear Algebra Appl., vol. 19, no. 4, pp. 639-654, 2012.
[5] H. Diao, X. Shi, and Y. Wei, "Effective condition numbers and small sample statistical condition estimation for the generalized Sylvester equation," Sci. China Math., vol. 56, no. 5, pp. 967-982, 2013.

[6] Y. Lin and Y. Wei, "Condition numbers of the generalized Sylvester equation," IEEE Trans. Autom. Contr., vol. 52, no. 12, pp. 2380-2385, 2007.

[7] Y. Lin, L. Bao, and Y. Wei, "Matrix sign function methods for solving projected generalized continuous-time Sylvester equations," IEEE Trans. Autom. Contr., vol. 55, no. 11, pp. 2629-2634, 2010.

[8] R.H. Bartels and G.W. Stewart, "Solution of the matrix equation AX+ $\mathrm{XB}=\mathrm{C}$," Commun. ACM. vol. 15, no. 9, pp. 820-826, 1972,.

[9] G. Golub, S. Nash, and C. Van Loan, "A Hessenberg-Schur method for the problem $\mathrm{AX}+\mathrm{XB}=\mathrm{C}$," IEEE Trans. Autom. Contr., vol. 24, no. 6 , pp. 909-913, 1979.

[10] B. Zhou, G. Duan, and Z. Li, "Gradient based iterative algorithm for solving coupled matrix equations," Syst. Contr. Lett., vol. 58, no. 5, pp. 327-333, 2009.

[11] L. Xie, Y. Liu, and H. Yang, "Gradient based and least squares based iterative algorithms for matrix equations $A X B+C X^{T} D=F$," Appl. Math. Comput., vol. 217, no. 5, pp. 2191-2199, 2010.

[12] F. Ding and T. Chen, "Gradient based iterative algorithms for solving a class of matrix equations," IEEE Trans. Autom. Contr., vol. 50, no. 8, pp. 1216-1221, 2005.

[13] F. Ding and H. Zhang, "Gradient-based iterative algorithm for a class of the coupled matrix equations related to control systems," IET Contr. Theor. Appl., vol. 8, no. 15, pp. 1588-1595, 2014.

[14] C. Song, J. Feng, X. Wang, and J. Zhao, "Finite iterative method for solving coupled Sylvester-transpose matrix equations," J. Appl. Math. Comput., vol. 46, no. 1-2, pp. 351-372, 2014.

[15] L. Xiao, B. Liao, S. Li, Z. Zhang, L. Ding, and L. Jin, "Design and analysis of FTZNN applied to the real-time solution of a nonstationary Lyapunov equation and tracking control of a wheeled mobile manipulator," IEEE Trans. Ind. Inform., vol. 14, no.1, pp. 98-105, 2018.

[16] Y. Xia and J. Wang "A bi-projection neural network for solving constrained quadratic optimization problems," IEEE Trans. Neural Netw. Learning Syst., vol. 27, no. 2, pp. 214-224, 2016.

[17] Z. Zhang, L. Zheng, J. Weng, Y. Mao, W. Lu, and L. Xiao, "A new varying-parameter recurrent neural-network for online solution of timevarying Sylvester equation," IEEE Trans. Cybern., vol. 48, no. 11, pp. 3135-3148, 2018.

[18] S. Li, Y. Zhang, and L. Jin, "Kinematic control of redundant manipulators using neural networks," IEEE Trans. Neural Netw. Learning Syst., vol. 28, no.10, pp. 2243-2254, 2017.

[19] L. Xiao, Z. Zhang, Z. Zhang, W. Li, and S. Li, "Design, verification and robotic application of a novel recurrent neural network for computing dynamic Sylvester equation," Neural Netw., vol. 105, pp. 185-196, 2018.

[20] L. Xiao, "A finite-time recurrent neural network for solving online timevarying Sylvester matrix equation based on a new evolution formula," Nonlin. Dyn., vol. 90, no. 3, pp. 1581-1591, 2017.

[21] S. Li, S. Chen, and B. Liu, "Accelerating a recurrent neural network to finite-time convergence for solving time-varying Sylvester equation by using a sign-bi-power activation function," Neural Process. Lett., vol. 37, no. 2, pp. 189-205, 2013.

[22] Y. Shen, P. Miao, Y. Huang, and Y. Shen, "Finite-time stability and its application for solving time-varying Sylvester equation by recurrent neural network," Neural Process. Lett., vol. 42, no. 3, pp. 763-784, 2015.

[23] S. Li and Y. Li "Nonlinearly activated neural network for solving timevarying complex Sylvester equation," IEEE Trans. Cybern., vol. 44, no. 8, pp. 1397-1407, 2014.

[24] P. S. Stanimirović, I. Živković, and Y. Wei, "Recurrent neural network approach based on the integral representation of the Drazin inverse," Neural Comput., vol. 27, no. 10, pp. 2107-2131, Oct. 2015.

[25] L. Xiao, "A nonlinearly-activated neurodynamic model and its finite-time solution to equality-constrained quadratic optimization with nonstationary coefficients," Appl. Soft Comput., vol. 40, pp. 252-259, 2016.

[26] H. Lu, L. Jin, X. Luo, B. Liao, D. Guo, and L. Xiao, "RNN for solving perturbed time-varying underdetermined linear system with double bound limits on residual errors and state variables," IEEE Trans. Ind. Informat., 2019, to be published.

[27] P. S. Stanimirović, I. Živković, and Y. Wei, "Recurrent neural network for computing the Drazin inverse," IEEE Trans. Neural Netw. Learning Syst., vol. 26, no. 11, pp. 2830-2843, Nov. 2015.

[28] M. Di Marco, M. Forti, P. Nistri, and L. Pancioni, "Discontinuous neural networks for finite-time solution of time-dependent linear equations," IEEE Trans. Cybern., vol. 46, no. 11, pp. 2509-2520, 2016. 
[29] L. Xiao, B. Liao, S. Li, and K. Chen, "Nonlinear recurrent neural networks for finite-time solution of general time-varying linear matrix equations," Neural Netw., vol. 98, pp. 102-113, 2018.

[30] S. Qiao, X. Wang, and Y. Wei, "Two finite-time convergent Zhang neural network models for time-varying complex matrix Drazin inverse," Linear Algebra Appl., vol. 542, pp. 101-117, 2018.

[31] H. Zhao, L. Li, H. Peng, J. Kurths, J. Xiao, and Y. Yang, "Finite-time robust synchronization of memrisive neural network with perturbation," Neural Process. Lett., vol. 47, no. 2, pp. 509-533, 2018.

[32] W. Li, "A recurrent neural network with explicitly definable convergence time for solving time-variant linear matrix equations," IEEE Trans. Ind. Informat., vol.14, no. 12, pp. 5289-5298, 2018.

[33] A. Polyakov, "Nonlinear feedback design for fixed-time stabilization of linear control systems," IEEE Trans. Autom. Contr., vol. 57, no. 8, pp. 2106-2110, 2012.

[34] A. Polyakov, D. Efimov, and W. Perruquetti, "Finite-time and fixed-time stabilization: Implicit Lyapunov function approach," Automatica, vol. 51, pp. 332-340, 2015.

[35] C. Chen, L. Li, H. Peng, and Y. Yang, "Fixed-time synchronization of inertial memristor-based neural networks with discrete delay," Neural Netw., vol. 109, pp. 81-89, 2019.

[36] W. Li, B. Liao, L. Xiao, and R. Lu, "A recurrent neural network with predefined-time convergence and improved noise tolerance for dynamic matrix square root finding," Neurocomputing, vol. 337,pp. 262-273, 2019.

[37] L. Jin, Y. Zhang, and S. Li, "Integration-enhanced Zhang neural network for real-time-varying matrix inversion in the presence of various kinds of noises," IEEE Trans. Neural Netw. Learning Syst., vol. 27, no. 12, pp. 2615-2627, 2016

[38] L. Jin, Y. Zhang, S. Li, and Y. Zhang, "Noise-tolerant ZNN models for solving time-varying zero-finding problems: A control-theoretic approach," IEEE Trans. Autom. Contr., vol. 62, no. 2, pp. 992-997, 2017.

[39] L. Jin, S. Li, L. Xiao, R. Lu, and B. Liao, "Cooperative motion generation in a distributed network of redundant robot manipulators with noises," IEEE Trans. Syst. Man Cybern. Syst., vol. 99, pp. 1-10, 2017.

[40] L. Jin, S. Li, B. Hu, M. Liu, and J. Yu, "Noise-suppressing neural algorithm for solving time-varying system of linear equations: A controlbased approach," IEEE Trans. Ind. Inform., vol. 15, no. 1, pp. 236-246, 2019.

[41] H. Zhao, L. Li, H. Peng, and Y. Wei, "A new approach of analyzing time-varying dynamical equation via an optimal principle," Mod. Phys. Lett. B, vol. 31, no. 8, pp. 1750084, 2018.

[42] M. Zheng, L. Li, H. Peng, J. Xiao, Y. Yang, and H. Zhao, "Parameters estimation and synchronization of uncertain coupling recurrent dynamical neural networks with time-varying delays based on adaptive control," Neural Comput. Appl., vol. 30, no. 7, pp. 81-89, 2018.

[43] D. Guo, Z. Nie, and L. Yan, "The application of noise-tolerant ZD design formula to robots' kinematic control via time-varying nonlinear equations solving," IEEE Trans. Syst. Man, Cybern. Syst., vol. 48, no. 12, pp. 2188-2197, 2018.

[44] Y. Zhang and S. S. Ge, "Design and analysis of a general recurrent neural network model for time-varying matrix inversion," IEEE Trans. Neural Netw., vol. 16, no. 6, pp. 1477-1490, 2005.

[45] L. Xiao, Y. Zhang, Z. Hu, and J. Dai, "Performance benefits of robust nonlinear zeroing neural network for finding accurate solution of lyapunov equation in presence of various noises," IEEE Trans. Ind. Informat., 2019, to be published.

[46] L. Xiao, "Design and analysis of robust nonlinear neural dynamics for solving dynamic nonlinear equation within finite time," Nonlinear Dyn., vol. 96, no. 4, pp. 2437-2447, 2019.

[47] B. Qiu, Y. Zhang, and Z. Yang, "New discrete-time ZNN models for least-squares solution of dynamic linear equation system with timevarying rank-deficient coefficient,", IEEE Trans. Neural Netw. Learning Syst., vol. 29, no. 11, pp. 5767-5776, 2018. 